\title{
Mitteilungen
}

Fähigkeitsausweis

\section{Prüfung Fähigkeitsausweis}

Klinische Notfallmedizin SGNOR 2019

Mündliche Prüfung (deutsch)

Ort: Bern Inselspital oder Bern Inselspital und Zürich Triemlispital*

Datum: 18. November 2019

Praktische Prüfung (deutsch)

Ort: Bern Sanitätspolizei oder Bern Sanitätspolizei und Zürich Triemlispital*

Datum: 25. November 2019

* bei mehr als 30 Kandidatinnen und Kandidaten wird die Prüfung parallel in Bern und Zürich durchgeführt

Anmeldefrist: 15. August 2019

Kosten: CHF 600 für Mitglieder SGNOR / CHF 1000 für Nichtmitglieder

Weitere Informationen finden Sie auf der Homepage der SGNOR www.sgnor.ch Fähigkeitsausweise - FA Klinische Notfallmedizin oder erhalten Sie bei der Geschäftsstelle der SGNOR: sekretariat[at]sgnor.ch

\section{Schweizerische Diabetes-Stiftung}

\section{Forschungspreis 2019}

Fachgebiet: Diabetologie

Arbeiten: Wissenschaftliche Arbeiten aus der Schweiz, die einen ausserordentlichen Beitrag darstellen an

- die Aufklärung der Ursachen
- die medizinischen oder sozialen Folgen

- die Verbesserung von Vorbeugung und Behandlung des Diabetes mellitus

\section{Preissumme: CHF 10000}

Teilnehmer: NachwuchsforscherInnen (Höchstalter 45 Jahre), die in den vergangenen zwei Jahren eine bedeutende diabetologische Arbeit publiziert haben

Eingabeformalitäten:

- Arbeiten und Publikationen aus den Jahren 2018 und 2019

- Curriculum Vitae inkl. Publikationsliste

- Die Arbeit darf nicht gleichzeitig für einen anderen Preis eingereicht werden oder bereits ausgezeichnet sein

- Co-Autoren müssen über die Eingabe informiert sein

- schriftlich oder elektronisch (PDF-Dateien)

- Zusammenfassung der Arbeit auf einer A4-Seite in Deutsch und Französisch

_ weitere Informationen: Tel. 0562001790

Eingabetermin: 1. September 2019

Schweizerische Diabetes-Stiftung,

Doris Fischer-Taeschler, Rütistrasse 3a, 5400 Baden, info[at]diabetesstiftung.ch

Preisverleihung: Jahresversammlung der Schweizerischen Gesellschaft für Endokrinologie und Diabetologie am 14. November 2019 in Bern

Jury: Stiftungsrat der Schweizerischen Diabetes-Stiftung
Nationale Ethikkommission im Bereich der Humanmedizin NEK

Zur Praxis des Abbruchs im späteren Verlauf der Schwangerschaft: NEK empfiehlt Massnahmen zur Sicherstellung einer hochstehenden und einheitlichen Versorgungsqualität

Abbrüche im späteren Verlauf der Schwangerschaft stellen alle Beteiligten vor grosse Herausforderungen, Unsicherheiten und Belastungen. Sie werfen zudem medizinische, rechtliche und ethische Fragen auf, die in der Schweiz bislang kaum thematisiert wurden.

Die Nationale Ethikkommission im Bereich der Humanmedizin (NEK) äussert sich dazu in ihrer jüngsten Stellungnahme. Von insgesamt über 10000 Schwangerschaftsabbrüchen, die in der Schweiz jährlich durchgeführt werden, finden knapp 500 Abbrüche nach der zwölften Schwangerschaftswoche statt. Rund 150 Abbrüche pro Jahr erfolgen in der weit fortgeschrittenen Schwangerschaft ab der 17. Schwangerschaftswoche. Rund 40 Abbrüche davon werden in der 23. Schwangerschaftswoche oder später vorgenommen. Ausgangspunkt ist stets eine Notlage der schwangeren Frau, die ärztlich bestätigt werden muss.

Bis zu welchem Zeitpunkt und in welchen Konstellationen Abbrüche im späteren Verlauf der Schwangerschaft vorgenommen werden, unterscheidet sich in der Schweiz von Klinik zu Klinik. In Bezug auf weit fortgeschrittene Schwangerschaften ist - je nach Region - der Zugang zu Abbrüchen erschwert; einzelne Kliniken führen daher überproportional viele solcher Eingriffe durch. 
Die Lebenssituation, die einem Abbruch bei fortgeschrittener Schwangerschaft zugrunde liegt, die daraus entstehende Entscheidungssituation und deren Folgen können betroffene Frauen und ihre Familien nachhaltig erschüttern. In der bestehenden Praxis werden Frauen zum Teil professionell unterstützt; teilweise sind die Begleitungsund Betreuungsangebote aber auch ungenügend koordiniert oder bestehen nicht über alle prä- und postnatalen Prozessphasen hinweg.

Ein Abbruch im späteren Verlauf der Schwangerschaft erfolgt hierzulande in der Regel durch eine medikamentös eingeleitete Geburt. Ab ungefähr der 17. Schwangerschaftswoche können Kinder, die infolge eines Abbruchs geboren werden, Lebenszeichen zeigen; ab etwa der 22. Schwangerschaftswoche ist mit intensivmedizinischer Unterstützung unter Umständen ein Überleben möglich. Kommt ein Kind nach einem Abbruch lebend zur Welt, wie dies in den Schweizer Neonatologie-Abteilungen jährlich in etwa 25 Fällen geschieht, gelten für seine Behandlung dieselben Kriterien wie bei anderen extrem frühgeborenen Kindern. Im Vordergrund steht eine gute palliative Betreuung. Abbrüche in der weit fortgeschrittenen Schwangerschaft werfen auch Fragen nach einer angemessenen Abbruchmethode auf.

In ihrer Stellungnahme untersucht die NEK die Praxis von Abbrüchen im späteren Verlauf der Schwangerschaft und setzt sich mit den komplexen medizinischen, rechtlichen und ethischen Fragen auseinander, die dadurch aufgeworfen werden. Auf dieser Grundlage formuliert sie Empfehlungen zur Versorgungssicherheit und zu Qualitätsstandards, zur Begleitung und Betreuung der schwangeren Frauen, zu den Methoden des Abbruchs und zum Umgang mit Lebendgeburten infolge eines Abbruchs. Zur Sicherstellung einer qualitativ hoch- und gleichwertigen Versorgung in der ganzen Schweiz empfiehlt die Kommission Massnahmen im Bereich des fachlichen Informations- und
Erfahrungsaustausches sowie die Erarbeitung und Verbreitung von Verfahrensstandards. Eine weitere zentrale Forderung besteht darin, dass betroffene Frauen umfassend über die verschiedenen Abbruchmethoden sowie die Alternativen zum Abbruch informiert und während des gesamten Prozesses (vor, während und nach dem Abbruch) kontinuierlich begleitet werden. Schliesslich hält die NEK in ihrer Stellungnahme fest, dass jedes Kind, das nach einem Schwangerschaftsabbruch lebend zur Welt kommt, medizinisch und pflegerisch umfassend versorgt und seine Lebenszeit würdig gestaltet werden muss. Es ist zudem sicherzustellen, dass die Frauen bzw. Paare über die Möglichkeit einer Lebendgeburt informiert werden, und dass das weitere Vorgehen für diesen Fall gemeinsam mit ihnen vorbesprochen wird.

Die Stellungnahme ist zu finden unter www.nek-cne.ch $\rightarrow$ Publikationen.

\footnotetext{
Aktuelle Themen auf unserer Website
www.saez.ch $\rightarrow$ tour d'horizon

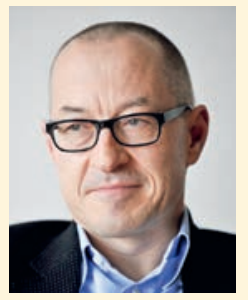

Interview mit David Bosshart, Leiter des Gottlieb Duttweiler Instituts «Was wir brauchen, sind Besserkönner, nicht Besserwisser»

Weshalb nur ein tiefgreifender Kulturwandel ein Ende der Kostenexplosion im Gesundheitswesen herbeiführen kann.

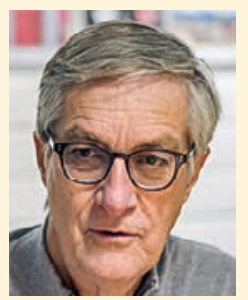

Interview mit Bernhard Keller, pensionierter Hausarzt und Betreiber der Plattform Rent-A-Senior-Doc

\section{«Hören wir doch auf zu jammern»}

Entwicklungen im Arbeitsmarkt wie beispielsweise der Wunsch nach einer WorkLife-Balance machen auch vor der Medizin nicht halt. Anstatt solche Trends zu kritisieren, kann man auch mit innovativen Konzepten darauf reagieren.
} 